IdeAs

Idées d'Amériques

18 | 2021

Frontières dans les Amériques - Intégration, sécurité et migrations

\title{
William R. Keylor, Charles de Gaulle, A Thorn in the Side of Six American Presidents
}

Lanham \& Londres, Rowman \& Littlefield

Isabelle Vagnoux

\section{OpenEdition \\ Journals}

Édition électronique

URL : https://journals.openedition.org/ideas/11863

DOI : 10.4000/ideas. 11863

ISSN : $1950-5701$

Éditeur

Institut des Amériques

Référence électronique

Isabelle Vagnoux, "William R. Keylor, Charles de Gaulle, A Thorn in the Side of Six American Presidents », IdeAs [En ligne], 18 | 2021, mis en ligne le 01 octobre 2021, consulté le 21 octobre 2021. URL : http:// journals.openedition.org/ideas/11863; DOI : https://doi.org/10.4000/ideas.11863

Ce document a été généré automatiquement le 21 octobre 2021.

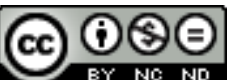

IdeAs - Idées d'Amériques est mis à disposition selon les termes de la licence Creative Commons Attribution - Pas d'Utilisation Commerciale - Pas de Modification 4.0 International. 


\title{
William R. Keylor, Charles de Gaulle, A Thorn in the Side of Six American Presidents
}

\author{
Lanham \& Londres, Rowman \& Littlefield
}

Isabelle Vagnoux

\section{RÉFÉRENCE}

William R. Keylor, Charles de Gaulle, A Thorn in the Side of Six American Presidents, Lanham \& Londres, Rowman \& Littlefield, 2020, 365 pages.

1 "Entre nous et les Américains, c'est la lutte », confiait le général de Gaulle à Alain Peyrefitte le 9 décembre 1964. C'est précisément cette "lutte» que l'historien américain William R. Keylor analyse, avec finesse et brio, dans son dernier ouvrage Charles de Gaulle, A Thorn in the Side of Six American Presidents, un de Gaulle jugé un peu "perdu» dès lors qu'il s'agissait de traiter avec les États-Unis pour lesquels il ne trouvait pas de « clés historiques » (257).

Dans un texte dense, concis et nuancé, élaboré à partir d'une riche bibliographie de sources secondaires et de très nombreuses archives, puisées des deux côtés de l'Atlantique, l'historien retrace la relation complexe que le général de Gaulle a entretenue avec cinq des six présidents américains auxquels il a eu affaire, de Franklin D. Roosevelt à Lyndon B. Johnson, avant de connaître une réelle embellie avec Richard M. Nixon.

W. Keylor s'attache systématiquement à souligner les divergences d'opinion au sein de chaque gouvernement, mais aussi les revirements des uns ou des autres, et les réactions de la presse. Les réticences de Roosevelt à soutenir la toute jeune et hardie France libre, pourtant considérablement aidée par l'allié Winston Churchill, apparaissent ainsi renforcées par la fidélité au régime de Vichy d'une bonne partie de la diaspora française aux États-Unis, la dissidence d'anciens militaires ayant participé aux 
premières heures du mouvement, et les désaccords entre de Gaulle et Jean Monnet, qui jouissait d'une belle influence au sein de l'Administration américaine. Alors que le département d'État est vent debout contre l'orgueilleux Général, le département de la Guerre apparaît quant à lui moins hostile mais demeure trop isolé au sein de l'Administration démocrate pour conduire à une relation bilatérale plus chaleureuse. Pire, en 1943, Roosevelt se dit convaincu que le général « a miné et continue de miner notre effort de guerre et qu'il représente pour nous un véritable danger. Il est grand temps de rompre avec lui » (73). De fait, l'alliance anglo-américaine resserre les rangs, à telle enseigne que même Churchill, soutien de la première heure, n'informe de Gaulle du débarquement allié en Normandie que trois jours avant D-day. L'insulte suprême vient avec la volonté délibérée de ne pas le convier à la conférence de Yalta. Truman fera de même quelques mois plus tard à la conférence de Potsdam. L'antipathie constante de Roosevelt à l'égard de de Gaulle et de la France libre, la froideur de Truman, teintée d'une vague francophobie, alliées au «nationalisme ardent» (258) du Général façonneront la politique étrangère du futur président et son désir viscéral de maintenir l'indépendance de la France à l'égard de Washington (259).

Une expérience commune d'anciens combattants, une réelle estime, voire admiration du Français pour le général Eisenhower à la tête du commandement américain en Europe, ainsi que sa reconnaissance pour son soutien face à une Administration Roosevelt très hostile, amènent une relation de travail « correcte » (259), voire cordiale, entre les deux hommes lorsque de Gaulle revient au pouvoir en 1958. Celui-ci affirme ainsi une solidarité sans faille face à Khrouchtchev («je suis avec vous jusqu'au bout » dira-t-il à Eisenhower (167) en 1960). De son côté, ce dernier soutient tacitement la politique française en Algérie. Contrairement à ses successeurs, le président américain comprend le désir français de posséder sa propre force nucléaire mais reste dans l'ambiguïté, face à un Congrès et un National Security Council hostiles à toute expansion du club très fermé des puissances nucléaires. En revanche, l'insistance gaullienne à mettre en place un directoire tripartite (États-Unis, Grande-Bretagne, France) au sein d'une OTAN dont les missions deviendraient "globales " parait "inacceptable » à Washington (159). Les deux dossiers, évoqués à chaque rencontre bilatérale, deviennent vite « un dialogue de sourds » (174).

5 Beaucoup plus fraîches seront les relations avec le démocrate John F. Kennedy, même si W. Keylor dépeint un Général sous le charme du jeune couple, qu'il accueille fastueusement à Paris en 1961. Toutefois entre les deux hommes, la lune de miel est de très courte durée et la tension apparaît particulièrement forte sur deux dossiers : d'une part, la force de frappe nucléaire française, véritable obsession gaullienne, alors que Kennedy insiste sur une Europe défendue par une Force nucléaire multilatérale menée par les Américains et l'OTAN; et d'autre part, l'entrée du Royaume-Uni dans la Communauté économique européenne, soutenue par Washington et vigoureusement rejetée par de Gaulle qui y voit un «cheval de Troie » et une tentative de domination des «Anglo-Saxons », ce que semble confirmer le Secrétaire d'État Dean Rusk dans ses mémoires (200). Deux visions de l'Europe s'affrontent: un bloc européen, incluant la Grande-Bretagne, étroitement arrimé à Washington (l'Alliance atlantique), option solidement défendue par l'influent George Ball ; ou bien une Europe continentale, alliée des États-Unis mais indépendante qui, sous la houlette de la France, développerait sa propre défense. En dépit de ce bras de fer, de Gaulle n'hésitera pas à accorder son plein 
soutien à Kennedy lors de la crise des missiles à Cuba, en 1962. Allié, mais nullement vassal...

6 La présidence Johnson représente sans doute le point culminant des tensions entre Paris et Washington, exacerbées par un Secrétaire d'État, Dean Rusk, déjà en poste sous Kennedy, qui ne ressent que dédain pour le président français. Les deux présidents ne se comprennent pas, s'agacent mutuellement (206) et sont en désaccord sur tout : sur le Vietnam pour lequel de Gaulle martèle qu'il doit être neutralisé grâce à un modus vivendi des nations asiatiques (211) alors que l'administration Johnson s'en tient à la théorie des dominos; sur la place de la Chine «rouge » que de Gaulle a décidé de reconnaître ; sur le nucléaire français ; ou encore sur l'OTAN, dont la France sortira. Si W. Keylor évoque en outre le désaccord sur l'intervention américaine en République dominicaine, il ne fait cependant pas mention du voyage de De Gaulle en Amérique latine en 1964, autre "irritant » dans une relation bilatérale déjà particulièrement tendue. Chose rare, en dehors de brefs échanges à l'occasion des funérailles de Kennedy et d'Adenauer, les deux présidents ne se sont jamais rencontrés. De Gaulle ne répondra pas favorablement à la proposition de la Maison-Blanche de mettre en place des conversations téléphoniques régulières, et malgré le soutien monétaire de Washington au franc, les Américains ne s'attendent à aucun effort de la part du président français. En fin de mandat, Johnson reconnaitra «ne pas avoir beaucoup fait personnellement pour améliorer cette situation ", sans avoir pour autant " contribué à sa détérioration » (246-247).

7 Il faudra attendre l'élection de Richard Nixon, converti au réalisme géopolitique après une carrière dominée par un anticommunisme fervent, pour que pointe enfin une « histoire d'amour » (261) bilatérale, certes brève, avant la démission du Général. Tous deux s'accordent à privilégier les intérêts de l'État-nation plutôt qu'une idéologie par définition passagère. La fin de la guerre du Vietnam - au demeurant plus longue que l'engagement américain sous Johnson (261) - la détente avec l'URSS, le début de la normalisation des relations avec Pékin, piliers de la politique de Nixon et Kissinger, avaient été préconisés par de Gaulle depuis des années.

8 Très agréable à lire, ponctuée de citations savoureuses qui rendent l'analyse historique particulièrement vivante, cette étude fait la part belle aux relations humaines susceptibles d'influer sur les considérations strictement géopolitiques. Certes, A Thorn in the Side of Six American Presidents est loin d'être le seul ouvrage, de part et d'autre de l'Atlantique, à traiter des relations franco-américaines pendant cette période, mais il est sans doute l'un des plus complets et des plus fouillés. À l'heure de la nouvelle alliance ("anglo-saxonne», selon la terminologie gaullienne) AUKUS, la lecture de Charles de Gaulle, A Thorn in the Side of Six American Presidents permet une belle mise en perspective historique des relations franco-américaines. 


\section{AUTEURS}

\section{ISABELLE VAGNOUX}

Rédactrice-en-chef d'IdeAs, Idées d'Amérique, Isabelle Vagnoux est professeur des Universités à Aix Marseille Univ, spécialiste de politique étrangère américaine et des relations avec l'Amérique latine. LERMA, UR 853

Isabelle.vagnoux@univ-amu.fr 